\title{
A Review of Bandwidth for Pediatric ECGs
}

\author{
Shen Luo ${ }^{1}$, Hong $\mathrm{Wei}^{1}$, Peter Macfarlane ${ }^{2}$ \\ ${ }^{1}$ StarCare Innovations Co, Ltd, Shenzhen, China \\ ${ }^{2}$ Institute of Health and Wellbeing, University of Glasgow, Glasgow, UK
}

\begin{abstract}
ECGs from neonates are known to have a higher frequency content than adult ECGs.

The aim of the study was to determine the effect of using different filter bandwidths on neonatal ECGs initially sampled at a rate of 8000 samples per second (which permits the use of a signal bandwidth much higher than $150 \mathrm{~Hz}$ ) and to consider the implications for routine ECG recording.

48 ECGs were recorded from newly born term infants (0-48 hours postnatal) at Princess Royal Maternity Hospital, Glasgow on a Burdick 8500 electrocardiograph. The frequency response of the machine was carefully checked. Peak to peak QRS amplitudes of average beats of the 10 second recordings were measured in all 8 independent leads with the results obtained at the full bandwidth of the ECG machine regarded as the reference.

The full bandwidth of the 8500 was verified as $0.05-$ $540 \mathrm{~Hz}$. It was found that the recommended upper frequency cutoff of $250 \mathrm{~Hz}$ in the current guideline does not meet the goal of amplitude errors $<25 \mu \mathrm{V}$ in $>95 \%$ of the cases in this data set. The clinical significance of high frequency components in pediatric ECGs is currently unclear.
\end{abstract}

\section{Introduction}

ECGs from neonates are known to have a higher frequency content than adult ECGs [1-3]. The 2007 AHA/ACC/HRS statement recommended an extension of the high-frequency cutoff to $250 \mathrm{~Hz}$ in children [4].

Higher frequency components may have clinical implications in adults [4-7], but there is still no convincing evidence to support the view that a diagnostic electrocardiograph clinically needs a bandwidth greater than $0.05-150 \mathrm{~Hz}$ particularly for neonatal ECG recording.

The aim of the study was to determine the effect of using different filtering bandwidths on infant
ECGs initially sampled at a rate of 8000 samples per second (sps), which permits the use of a signal bandwidth much higher than $150 \mathrm{~Hz}$ as well as 250 $\mathrm{Hz}$, and to consider the implications for routine ECG recording.

\section{Methods}

The normal neonatal QRS amplitude is maximal during the first day or two of life and then gradually decreases for a very short period before increasing over the years as the child grows [2]. In the study, therefore, 48 ECGs were recorded from newly born term infants, who were $0-48$ hours postnatal, at the Princess Royal Maternity Hospital, Glasgow.

A Burdick 8500 electrocardiograph was used. All 8 independent leads, I, II, V1 - V6 with V4R replacing V3, were sampled at $8000 \mathrm{sps}$. The high cutoff frequency of the machine, which is $540 \mathrm{~Hz}$ ($3 \mathrm{~dB}$ ), was confirmed by using the testing method per ANSI/AAMI EC11 [8]. The frequency data and the 8-lead infant ECGs, both at 8000 sps, were transferred to a PC for frequency response checks and further data processing and analysis.

48 pediatric ECGs were processed by a specially developed computer algorithm. All pediatric ECG beats were first identified.

It is generally assumed that an averaged 10 second ECG waveform will only reduce the noise without changing the true ECG waveform itself. After an average (representative) beat for each lead was produced from 10-second recordings through both detected fiducial points and cross-correlation methods (Window width: 500 samples at 8000 sps or $62.5 \mathrm{~ms}$ ) for alignment (Figures 1 and 2), the peak to peak QRS amplitudes in these leads were measured. They were regarded as the reference amplitudes for comparison thereafter. 


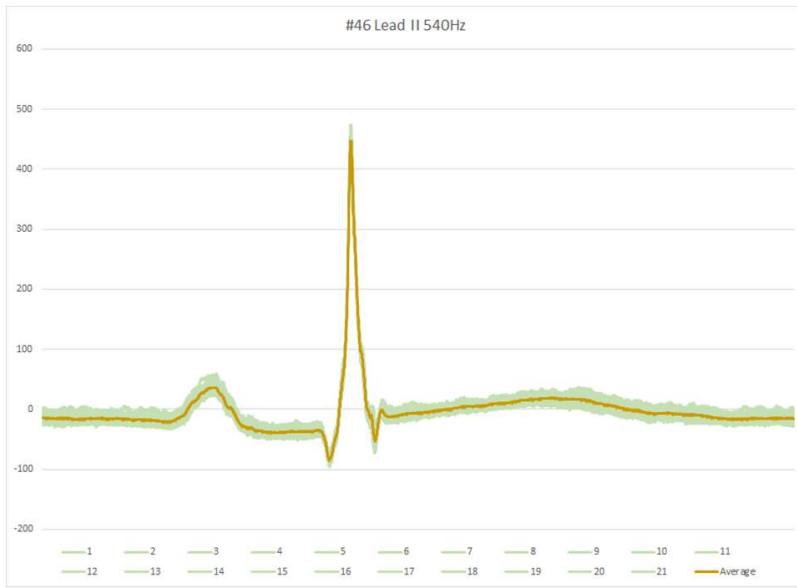

Figure 1 Overlay display of 21 aligned raw beats and the average beat for a 10 -second period in Lead II from Infant \#46.

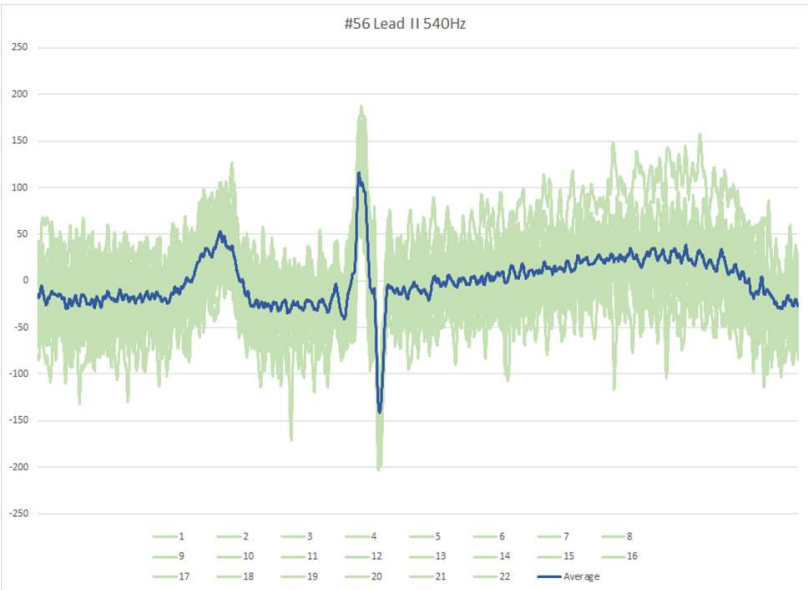

Figure 2 Overlay display of 22 aligned raw beats and the average beat for 10 -second period in Lead II from Infant \#56.

The raw signals of $0.05-540 \mathrm{~Hz}$ were also processed by $250 \mathrm{~Hz}$ and $150 \mathrm{~Hz}$ FIR low-pass filters at 8000 sps separately (Figure 3 and 4). After that, a similar procedure for the full bandwidth above was then applied to each group of the filtered data to obtain averaged beat and peak to peak QRS amplitudes for each filtered lead.

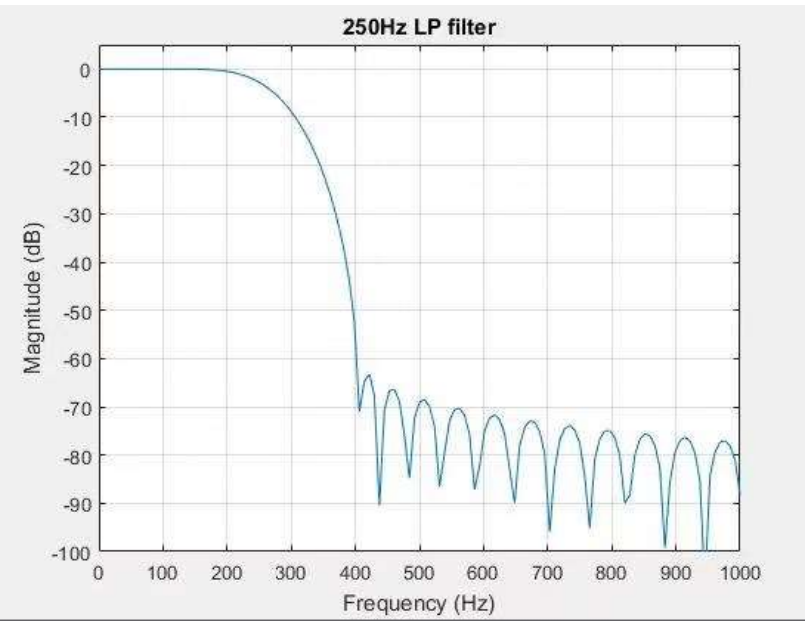

Figure 3 The frequency response of the $250-\mathrm{Hz}$ FIR lowpass filter with 65 taps at 8000 sps.

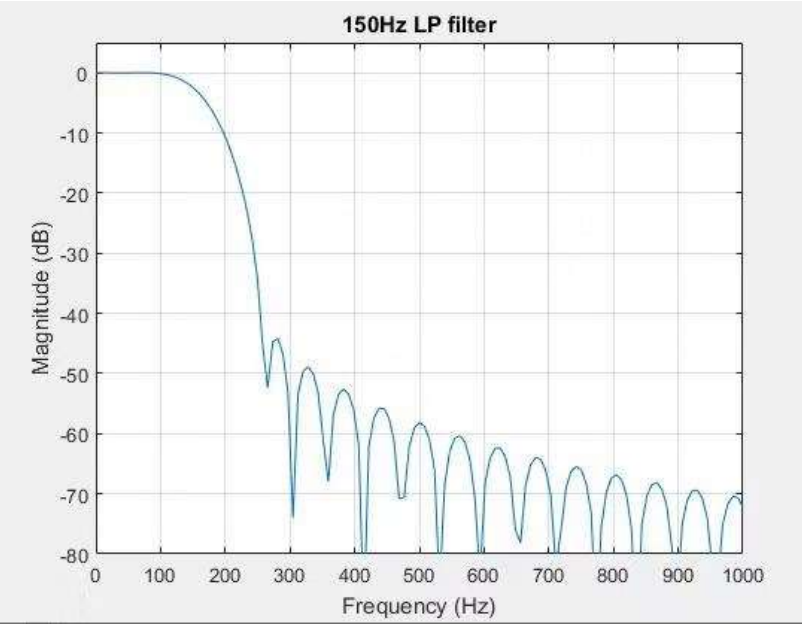

Figure 4 The frequency response of the 150-Hz FIR lowpass filter with 65 taps at 8000 sps.

Finally, the differences between the reference amplitudes and the specific filtered amplitudes for each lead were calculated. All the outcomes, including average waveforms and measurement points, were validated by two clinical technicians.

\section{Results}

The 48 infant ECGs with full bandwidth (reference) across 8 independent leads had typically 
short QRS durations with a range from 37.5 to $66 \mathrm{~ms}$, and very high QRS amplitudes up to $6.9 \mathrm{mV}$.

The higher frequency components of ECGs in the frequency domain are simply related to narrow $\mathrm{QRS}$ complexes and high QRS amplitudes with detailed notches in the time domain. Reducing upper cutoff frequency can basically be implemented by using low-pass filters on the data, which will not change the QRS durations if the process is properly achieved but will reduce the amplitudes and smooth notches somewhat.

The goal from the recommendation is amplitude errors $<25 \mu \mathrm{V}$ in $>95 \%$ cases [3-4]. For a simulated bandwidth of $250 \mathrm{~Hz}, 29$ out of 384 cases, that is $7.6 \%$, of the QRS amplitude differences from the reference were greater than $25 \mu \mathrm{V}$. The maximum difference was $49.3 \mu \mathrm{V}$. Table 1 shows how many QRS amplitude differences are more than $25 \mu \mathrm{V}$ for each individual lead. Except for Lead I and V4R, the other 6 leads (II, V1, V2, V4-V6) are from the $81.2^{\text {th }}$ to $93.7^{\text {th }}$ percentiles, not meeting the $95^{\text {th }}$ percentile requirement based on this infant data set.

Table 1. Number of leads and percentage of cases exceeding $25 \mu \mathrm{V}$ from reference values when bandwidth is $0.05-250 \mathrm{~Hz}$.

\begin{tabular}{|c|c|c|}
\hline Lead & $>\mathbf{2 5} \boldsymbol{\mu} \mathbf{V}$ & \% of $\mathbf{4 8}$ cases \\
\hline I & 0 & 0.0 \\
\hline II & 3 & 6.3 \\
\hline V4R & 1 & 2.1 \\
\hline V1 & 4 & 8.3 \\
\hline V2 & 9 & 18.8 \\
\hline V4 & 3 & 6.3 \\
\hline V5 & 5 & 10.4 \\
\hline V6 & 4 & 8.3 \\
\hline
\end{tabular}

For a simulated bandwidth of $150 \mathrm{~Hz}$, which is the bandwidth most commonly used for ECG recording, 116 out of 384 cases, that is $30.2 \%$, of the QRS amplitude differences from the reference were greater than $25 \mu \mathrm{V}$. Table 2 shows how many QRS amplitude differences of more than $25 \mu \mathrm{V}$ for each individual lead were found.

The maximum QRS amplitude difference compared to the reference and with $150-\mathrm{Hz}$ cutoff was found in Lead V2 of infant \#15.

However, the gross percentage of QRS amplitudes with $150 \mathrm{~Hz}$ cutoff meeting the reference was $99.08 \% \pm 1.37 \%$.
Table 3 shows the gross QRS amplitude.

Table 2. Number of leads and percentage of cases exceeding $25 \mu \mathrm{V}$ from reference values when bandwidth is $0.05-150 \mathrm{~Hz}$.

\begin{tabular}{|c|c|c|}
\hline Lead & $>\mathbf{2 5} \boldsymbol{\mu} \mathbf{V}$ & \% of $\mathbf{4 8}$ cases \\
\hline I & 3 & 6.3 \\
\hline II & 15 & 31.3 \\
\hline V4R & 9 & 18.8 \\
\hline V1 & 21 & 43.8 \\
\hline V2 & 22 & 45.8 \\
\hline V4 & 17 & 35.4 \\
\hline V5 & 15 & 31.3 \\
\hline V6 & 14 & 29.2 \\
\hline
\end{tabular}

Table 3. Mean and standard deviation of gross peak to peak QRS amplitude.

\begin{tabular}{|c|c|c|}
\hline Data & Mean $(\boldsymbol{\mu V})$ & StDev $(\boldsymbol{\mu V})$ \\
\hline $540 \mathrm{~Hz}(\mathrm{REF})$ & 2506.00 & \pm 1542.11 \\
\hline $250 \mathrm{~Hz}$ & 2498.66 & \pm 1540.99 \\
\hline $150 \mathrm{~Hz}$ & 2489.37 & \pm 1539.74 \\
\hline
\end{tabular}

\section{Discussion}

For infants, the recommendation of $250 \mathrm{~Hz}$ highfrequency cutoff still does not meet the goal of amplitude errors $<25 \mu \mathrm{V}$ in $>95 \%$ of the cases according to Table 1 from this data set. The original recommendation of a $250 \mathrm{~Hz}$ cutoff [3] is based on a $320 \mathrm{~Hz}$ reference amplifier cutoff that may not be high enough to evaluate the performances at $250 \mathrm{~Hz}$.

To verify the $250 \mathrm{~Hz}$ high-frequency cutoff for children, this study employed a device with a $540 \mathrm{~Hz}$ upper frequency cutoff, which is much higher than $320 \mathrm{~Hz}$ used in the research report. Further, the device used in the study has 8000 sps raw data rather than 1200 sps per lead. Technically, the significantly higher sampling rate will be able to adequately attenuate aliasing distortions [9].

Newborn ECG records usually have a lot of noise (Figure 2) with a random component. There are two main sources, one being that infants are constantly moving (it is impossible to tell them to stop!) and the 
other is that bandwidth is greatly increased. The averaging processing is necessary, otherwise, in many cases of a low signal-to-noise ratio, the attenuation does not completely represent the reduction of the true signal amplitude.

Table 3 shows that the absolute mean difference between the $150 \mathrm{~Hz}$ and the $540 \mathrm{~Hz}$ reference values is no greater than $17 \mu \mathrm{V}$ and the absolute standard deviation is no greater than $2.5 \mu \mathrm{V}$. The relative mean error, however, is only $0.66 \%$, and the relative standard deviation is $0.15 \%$. A question here is how Pediatric Cardiologists view, or would they care about, these "small" attenuations?

Further data analysis is needed.

In summary, the $250 \mathrm{~Hz}$ high-frequency cutoff still does not meet the goal of amplitude errors $<25 \mu \mathrm{V}$ in $>95 \%$ of the cases according to this data set. The clinical significance of these small attenuations even from $150 \mathrm{~Hz}$ data vs the reference is unclear.

\section{Acknowledgments}

Special thanks to Pei Wang for assistance in filter design.

\section{References}

[1] Liebman J, Plonsey R, Gillette PC (eds). "Pediatric Electrocardiography," Baltimore, Maryland, Williams and Wilkins. 1982.

[2] Macfarlane PW, Coleman E, Pomphrey EO et al. "Normal limits of the high-fidelity pediatric ECG: preliminary observations," J Electrocardiol 1989;22 (Suppl):162-168.

[3] Rijnbeek PR, Kors JA, Witsenburg J. "Minimum bandwidth requirements for recording of pediatric electrocardiograms," Circulation 2001; 104: 3087-3090.

[4] Kligfield P, Gettes LS, Bailey J, et al. "Recommendations for the standardization and interpretation of the electrocardiogram part I: The Electrocardiogram and Its Technology. A Scientific Statement from the American Heart Association Electrocardiography and Arrhythmias Committee, Council on Clinical Cardiology; the American College of Cardiology Foundation; and the Heart Rhythm Society," Circulation 2007; 115: 1306-24.
[5] Goldberger AL, Bhargava V, Froelicher V, Covell J. "Effect of myocardial infarction on high frequency QRS potentials," Circulation 1981; 64: 34-42.

[6] Pettersson J, Warren S, Mehta N, et al. "Changes in high-frequency QRS components during prolonged coronary artery occlusion in humans," J Electrocardiol 1995;28(Suppl):225-7.

[7] Petterson J, Carro E, Edenbrandt L, et al. "Spatial, individual, and temporal variation of the highfrequency QRS amplitudes in the 12 standard electrocardiographic leads," Am Heart J 2000; 139: 352-8.

[8] American National Standards Institute. "Diagnostic electrocardiographic devices (ANSI/AAMI EC11:1991/(R)2001(R)/2007)," Arlington, VA: Association for the Advancement of Medical Instrumentation, 2007

[9] Luo S, Johnston P. "A review of the electrocardiogram filtering," J Electrocardiol 2010; 43: 486-96

Address for correspondence:

Shen Luo, PhD

StarCare Innovations Co., Ltd, Shenzhen, China shenl@starcaretech.com 